\title{
Understanding the relationship between the inbreeding coefficient and multilocus heterozygosity: theoretical expectations and empirical data
}

\author{
J Slate $^{1}$, P David ${ }^{2}$, KG Dodds ${ }^{1}$, BA Veenvliet ${ }^{1}$, BC Glass $^{1}$, TE Broad ${ }^{1,3}$ and JC McEwan ${ }^{1}$ \\ ${ }^{1}$ AgResearch, Invermay Agricultural Centre, Mosgiel, Private Bag 50034, New Zealand; ${ }^{2}$ Centre d'Ecologie Fonctionelle et Evolutive, \\ Centre National de la Recherche Scientifique, 34293, Montpellier Cedex 5, France
}

\begin{abstract}
Geneticists have been interested in inbreeding and inbreeding depression since the time of Darwin. Two alternative approaches that can be used to measure how inbred an individual is involve the use of pedigree records to estimate inbreeding coefficients or molecular markers to measure multilocus heterozygosity. However, the relationship between inbreeding coefficient and heterozygosity has only rarely been investigated. In this paper, a framework to predict the relationship between the two variables is presented. In addition, microsatellite genotypes at 138 loci spanning all 26 autosomes of the sheep genome were used to investigate the relationship between inbreeding coefficient and multi-
\end{abstract}

locus heterozygosity. Multilocus heterozygosity was only weakly correlated with inbreeding coefficient, and heterozygosity was not positively correlated between markers more often than expected by chance. Inbreeding coefficient, but not multilocus heterozygosity, detected evidence of inbreeding depression for morphological traits. The relevance of these findings to the causes of heterozygosity-fitness correlations is discussed and predictions for other wild and captive populations are presented.

Heredity (2004) 93, 255-265. doi:10.1038/sj.hdy.6800485

Published online 14 July 2004

Keywords: inbreeding; heterosis; heterozygosity; overdominance; identity disequilibrium

\section{Introduction}

When related individuals mate, their offspring are generally less viable, less fertile or smaller than the population mean - a phenomenon known as inbreeding depression. Consequently, inbreeding has been the focus of considerable attention in a number of areas of biology including animal and crop production, human medicine, conservation biology and the evolution of mating systems (Thornhill, 1993; Hedrick and Kalinowski, 2000; Keller and Waller, 2002). Inbreeding depression arises because inbreeding increases the probability that an individual will be (a) homozygous for segregating deleterious recessive alleles and (b) homozygous at loci exhibiting overdominance (Falconer, 1989; Lynch and Walsh, 1998). Deleterious recessive alleles are thought to be the major cause of inbreeding depression (Charlesworth and Charlesworth, 1999).

Inbreeding depression can be inferred in a number of ways (Keller and Waller, 2002). The most straightforward approaches utilise the inbreeding coefficient $f$ (Wright, 1922) - defined as the probability that two alleles at a locus are identical by descent (ibd). For example, relatives can be deliberately mated to

Correspondence: J Slate, Current address: Department of Animal and Plant Sciences, University of Sheffield, Western Bank S10 3TN, UK.

E-mail: j.slate@sheffield.ac.uk

${ }^{3}$ Current address: Australian Equine Genetics Research Centre (AEGRC), University of Queensland, St Lucia, Brisbane, QLD 4072, Australia Received 27 September 2002; accepted 18 November 2003; published online 14 July 2004 produce individuals of known $f$, which are then compared to outbred individuals from the same population. A similar approach is to use pedigree records to calculate $f$ for all of the individuals in the population. Inbreeding depression is then inferred by regressing phenotype (or log-transformed phenotype) on the inbreeding coefficient (Morton et al, 1956; Lynch and Walsh, 1998).

If inbreeding coefficients are unavailable, an alternative approach is to examine the association between marker heterozygosity (typically measured at 5-10 loci) and phenotypic value. This approach, sometimes termed heterozygosity-fitness correlations (HFCS), originated with the advent of soluble allozyme markers (Allendorf and Leary, 1986; Mitton, 1993; David, 1998). Initial investigations with allozymes sought to address whether genetic variation was maintained by drift or selection; in other words, did genotype at individual loci have a direct effect on fitness? However, in recent years, a number of studies have reported significant relationships between multilocus heterozygosity (hereafter MLH) in noncoding DNA and fitness-related traits in wild populations, with inbreeding depression usually regarded as the most likely explanation for the relationship (Coltman et al, 1999; Marshall and Spalton, 2000; Slate et al, 2000; Amos et al, 2001; Acevedo-Whitehouse et al, 2003). This explanation is intuitively appealing as inbred individuals are expected to be relatively homozygous throughout the genome. However, the inbreeding coefficient and MLH do not measure the same quantity. When two alleles at a locus are ibd, the genotype is said to be 
autozygous, otherwise the genotype is allozygous. Allozygous genotypes may be homozygous (identical by state) or heterozygous, but in the absence of recent mutation an autozygous genotype is always homozygous (Hartl and Clark, 1997).

Unfortunately, there are (at least) three possible explanations for HFCs (David, 1998; Hansson and Westerberg, 2002), only one of which requires inbreeding. The first is that some or all of the marker loci have a direct effect on fitness with heterozygous genotypes conferring the greatest fitness (ie the locus exhibits overdominance). This explanation can generally be excluded for studies using microsatellite loci as they are usually nonfunctional. The second explanation is that the markers are in physical linkage with either overdominant or dominant loci that influence fitness. If a marker and trait locus are in linkage disequilibrium, then individuals that are heterozygous at the marker locus will also tend to be heterozygous at the trait locus. This explanation is often termed a local effect. A third explanation is that the heterozygosity at marker loci reflects heterozygosity at unlinked trait loci. Such an association is only expected to arise in populations that exhibit variance in inbreeding and is sometimes termed a general effect or identity disequilibrium (Weir and Cockerham, 1973). It is this explanation that is invoked when it is claimed that an HFC indicates inbreeding depression. Note that the first two explanations do not require inbreeding. Spurious HFCs can also arise when individuals are sampled from several populations that exhibit between-population variation in both heterozygosity and nongenetic (eg environmental) components of trait variance. In other words, the HFC is an artefact of population structure.

Given that $f$ and MLH are popular metrics for inferring inbreeding depression, it is surprising that the relationship between the two has only rarely been investigated theoretically (Bierne et al, 2000) or empirically (Hedrick et al, 2001; Curik et al, 2003). Further investigation of the relationship would not only help determine when MLH can be used as a reliable alternative to $f$, but would also help to ascertain the genetic basis of HFCs (Hansson and Westerberg, 2002). Indeed, most of the available HFC theory predicts that MLH and fitness, under the inbreeding hypothesis, should only correlate as a consequence of their common dependence on $f$. When only MLH and fitness are known, $f$ remains an implicit variable. But having an independent knowledge of $f$ from pedigrees allows one to test properly the central point of the theory. To our knowledge, no study combines estimates of heterozygosity at a large number of loci dispersed across the genome, accurate knowledge of $f$ using detailed pedigrees, and measures of phenotypic variation. Domestic livestock populations offer opportunities to address this problem because stud books are often available, and comprehensive genotype data sets are arising from genome-wide scans for quantitative trait loci (QTL).

The aims of this study were to

(i) predict the relationship between MLH and individual $f$ under a simple model whereby the expected heterozygosity at a marker locus is a function of $f$, and heterozygosity is uncorrelated across marker loci in individuals of the same $f$; (ii) test this theoretical prediction with an extensive data set (590 individuals of known $f$, typed at up to 138 microsatellites) from a domestic sheep population.

(iii) provide some predictions on the relationship between $f$ and MLH in several intensively studied populations, in order to determine how effectively microsatellites can be used to infer inbreeding depression in wild populations.

\section{Methods}

\section{The model}

It is assumed that the inbreeding level of an individual is characterised by a single $f$ value determined by its pedigree. All loci are assumed to be equally affected by inbreeding. Note that estimates of $f$ based on known pedigrees may slightly differ from the true value of $f$, as founders are assumed to have an $f$ of zero. However, provided the pedigrees are correct and several generations (three or more) deep, they should enable good estimates of true $f$. The distribution of $f$ has probability density function $p(f)$.

Let $h_{i}$ be the heterozygosity ( 0 for a homozygote and 1 for a heterozygote at marker locus i). The expected heterozygosity $E\left(h_{i}\right)$ at locus $i$ is $h_{0, i}(1-f)$ for a given $f$, where $h_{0, i}$ is the genetic diversity at locus $i$, or the expected heterozygosity in the absence of inbreeding.

It is assumed that any correlation among $h_{i}$ at different loci across individuals only arises due to within-population variation in $f$. In other words, no correlation will be expected in individuals that share the same value of $f$. This is usually true for unlinked loci. Note that $96 \%$ of locus pairs are unlinked in the Coopworth sheep data set presented in this study, with only $0.12 \%$ of locus pairs located within $10 \mathrm{cM}$ of each other.

The term $H$ refers to standardised MLH, calculated as the proportion of typed loci at which an individual was heterozygous divided by the population mean heterozygosity of those typed loci. This standardisation was initially used by Coltman et al (1999) in a study of Soay sheep, where individuals had been typed at different subsets of the same loci. The standardisation ensures that all individuals are measured on an identical scale and has subsequently been employed elsewhere (Slate et al, 2000; Amos et al, 2001). Standardised MLH is usually highly correlated with the more traditional nonstandardised version:

$$
H=\frac{\sum_{i} h_{i}}{\sum_{i} \bar{h}_{i}}
$$

where $\overline{h_{i}}$ is the mean heterozygosity of all individuals typed at locus $i$ and summation is across all typed loci.

The predicted distribution of heterozygosity: $f$ has distribution with mean $E(f)$ and variance $\sigma^{2}(f)$. We first derive the moments of the distribution of multiple-locus heterozygosity as functions $E(f)$ and $\sigma^{2}(f)$. Then we will use these expressions to derive the expected correlations between $f$ and heterozygosity, and between heterozygosity and fitness. 
From the relationship above, the expected heterozygosity at locus $i$ is

$$
\begin{aligned}
E\left(h_{i}\right) & =\int E\left(h_{i} \mid f\right) p(f) \mathrm{d} f \\
& =\int h_{0, i}(1-f) p(f) \mathrm{d} f=h_{0, i}(1-E(f))
\end{aligned}
$$

and, by definition, $E(H)=1$.

Given that $h_{i}$ can only take the values 0 or $1, h_{i}^{2}=h_{i}$, the variance in $h_{i}$ is

$$
\begin{aligned}
\sigma^{2}\left(h_{i}\right) & =E\left(h_{i}\right)-E\left(h_{i}\right)^{2} \\
& =h_{0, i}(1-E(f))-h_{0, i}^{2}(1-E(f))^{2}
\end{aligned}
$$

The covariance in heterozygosity among loci $i$ and $j$ is

$$
\sigma\left(h_{i}, h_{j}\right)=E\left(h_{i} h_{j}\right)-E\left(h_{i}\right) E\left(h_{j}\right)
$$

where

$$
\begin{aligned}
E\left(h_{i} h_{j}\right) & =\int E\left(h_{i} \mid f\right) E\left(h_{j} \mid f\right) p(f) \mathrm{d} f \\
& =h_{0, i} h_{0, j} \int(1-f) 2 p(f) \mathrm{d} f \\
& =h_{0, i} h_{0, j}\left(1-2 E(f)+E\left(f^{2}\right)\right)
\end{aligned}
$$

and

$$
\begin{aligned}
E\left(h_{i}\right) E\left(h_{j}\right) & =h_{0, i} h_{0, j}(1-E(f))^{2} \\
& =h_{0, i} h_{0, j}\left(1-2 E(f)-E(f)^{2}\right)
\end{aligned}
$$

which yields

$$
\begin{aligned}
\sigma\left(h_{i}, h_{j}\right) & =h_{0, i} h_{0, j}\left(E\left(f^{2}\right)-E(f)^{2}\right) \\
& =h_{0, i} h_{0, j} \sigma^{2}(f)
\end{aligned}
$$

The variance in $H$ is

$$
\begin{aligned}
\sigma^{2}(H) & =\left(1 / \sum_{i} E\left(h_{i}\right)\right)^{2} \sigma^{2}\left(\sum{ }_{i} h_{i}\right) \\
& =\frac{\left(1 / \sum_{i} E\left(h_{0, i}\right)\right)^{2} \sigma^{2}\left(\sum{ }_{i} h_{i}\right)}{(1-E(f))^{2}}
\end{aligned}
$$

where

$$
\begin{aligned}
\sigma^{2}\left(\sum_{i} h_{i}\right)= & \sum_{i} \sigma^{2}\left(h_{i}\right)+2 \sum_{i} \sum_{j} \sigma\left(h_{i}, h_{j}\right) \\
= & (1-E(f)) \sum_{i} h_{0, i}-(1-E(f))^{2} \\
& \times \sum{ }_{i} h_{0, i}^{2}+\sigma^{2}(f)^{2} \sum_{i} \sum_{j} h_{0, i} h_{0, j}
\end{aligned}
$$

Then, after some algebra, we obtain

$$
\begin{aligned}
\sigma^{2}(H)= & \frac{1}{(1-E(f)) \sum_{i} h_{0, i}}-\frac{\sum_{i} h_{0, i}^{2}}{\left(\sum_{i} h_{0, i}\right)^{2}} \\
& \times\left(1+\frac{\sigma^{2}(f)}{(1-E(f))^{2}}\right)+\frac{\sigma^{2}(f)}{(1-E(f))^{2}}
\end{aligned}
$$

This expression involves several terms that depend on intrinsic characteristics of the set of loci analysed (number of loci, genetic diversity and how it varies across loci). In order to increase clarity, we can consider a first-order approximation that makes the simplifying assumption that all loci have the same genetic diversity $h_{0}$. The expression for the variance in $H$ then becomes

$$
\begin{aligned}
\sigma^{2}(H)= & \frac{1}{(1-E(f)) h_{0} L}-\frac{1}{L}\left(1+\frac{\sigma^{2}(f)}{(1-E(f))^{2}}\right) \\
& +\frac{\sigma^{2}(f)}{(1-E(f))^{2}}
\end{aligned}
$$

where $L$ is the number of typed loci. It is apparent that, as the number of typed loci increases, the third term alone will be a good approximation of the variance in $H$, which will be roughly proportional to the variance in inbreeding.

The predicted correlation in heterozygosity between two loci: The correlation in heterozygosity between two loci is obtained using the above expressions for the covariance and variance in heterozygosity at loci $i$ and $j$ :

$$
\begin{aligned}
& r\left(h_{i}, h_{j}\right)= \\
& \frac{\sigma^{2}(f)}{1-E(f)) \sqrt{h_{0, i} h_{0, j}\left(1-h_{0, i}(1-E(f))\right)\left(1-h_{0, j}(1-E(f))\right)}}
\end{aligned}
$$

The standard error of the estimated $r\left(h_{i}, h_{j}\right)$ is (Zar, 1996)

$$
S_{r}=\sqrt{\frac{1-r_{h_{i}, h_{j}}^{2}}{n-2}}
$$

The predicted correlation between individual heterozygosity and inbreeding coefficient: The covariance between heterozygosity and $f$ is

$$
\sigma\left(h_{i}, f\right)=E\left(h_{i}, f\right)-E\left(h_{i}\right) E(f)
$$

The first term of this expression reduces to

$$
\begin{aligned}
E\left(h_{i} f\right) & =\int E\left(h_{i} \mid f\right) f p(f) \mathrm{d} f \\
& =\int h_{0, i}(1-f) f p(f) \mathrm{d} f=h_{0, i}\left(E(f)-E\left(f^{2}\right)\right)
\end{aligned}
$$

so that the covariance between heterozygosity and $f$ can be written as

$$
\begin{aligned}
\sigma\left(h_{i}, f\right) & =h_{0, i}\left(E(f)-E\left(f^{2}\right)\right)-h_{0, i}\left(E(f)-E(f)^{2}\right. \\
& =-h_{0, i} \sigma^{2}(f)
\end{aligned}
$$

The covariance between $H$ and $f$ is

$$
\sigma(H, f)=\frac{\sum_{i} \sigma\left(h_{i}, f\right)}{\sum_{i} E\left(h_{i}\right)}=\frac{-\sigma^{2}(f)}{(1-E(f))}
$$

and the correlation between $H$ and $f$ is

$$
r(H, f)=\frac{-\sigma^{2}(f)}{(1-E(f)) \sigma(f) \sigma(H)}=\frac{-\sigma(f)}{(1-E(f)) \sigma(H)}
$$

The predicted correlation between $\mathrm{H}$ and fitness traits: We assume the Morton et al (1956) model for the relationship between inbreeding and fitness traits such that the trait (or the logarithm of the trait) declines as a linear function of $f$. Thus, $W=a-b f$, where $W$ is the 
trait, $a$ is a constant and $b$ is the inbreeding load. $b$ can be estimated by linear regression of $W$ on $f$.

If we assume that all correlations between heterozygosity and the trait arise as a result of inbreeding, then

$$
r(W, H)=r(W, f) r(H, f)
$$

$r(W, f)$ is estimated by the regression of the trait on $f$, while $r(H, f)$ is defined above (equation (4)).

\section{Application of the model to a real data set}

Coopworth sheep were developed by crossing the Romney and Border Leicester breeds in New Zealand in the 1950s. The breed society was formally recognised in 1968 and Coopworths are now the second most numerous breed in New Zealand. We investigated a population of Coopworth sheep that was founded from six farms in the 1970s and has been the subject of divergent selection for backfat depth since 1981 (Morris et al, 1997). Subsequently, five F1 (fat $\times$ lean) sires have been backcrossed to both the fat and lean lines as part of an experiment to map QTL for morphological traits (Campbell et al, 2003). A total of 590 progeny were typed at up to 138 approximately evenly spaced microsatellite loci, spanning all 26 autosomes. Every individual was measured for a number of morphological traits and various potential explanatory variables were also recorded (see below). Inbreeding coefficients were calculated using the routine PROC INBREED, implemented in SAS (SAS Institute, Cary, NC, USA). For every individual, 7-10 generations of ancestors were known, dating back to the foundation of the selection lines, enabling accurate calculation of $f$. It was assumed that all founder individuals had an $f$ of zero and were unrelated. MLH at all 138 loci was calculated, and converted to standardised MLH (see Coltman et al (1999) and above). Hereafter, MLH refers to the standardised version $(H$ in the above model). Note that progeny were not genotyped at loci for which the sire was homozygous. Thus the genotype data file was only $73 \%$ complete - equivalent to an average of 101 genotypes per individual. The mean number of typed loci per half-sib family ranged from 98 (sire 603) to 106 (sires 610 and 616). In subsequent analyses, we report the expected relationship between $f$ and MLH assuming that 101 loci were typed.

These data were then used to address three questions:

(1) What is the relationship between $f$ and MLH?

(2) Is heterozygosity correlated between loci?

(3) Do either $f$ or MLH explain phenotypic variation?

The relationship between the two measures of inbreeding and 10 morphological traits (empty body weight, hot carcass weight, spleen weight, liver weight, heart weight, backfat depth at the 12th rib, tibia length, carcass length, longissimus dorsi weight and testes weight) was investigated. All traits appeared normally distributed (spleen weight was log-transformed), so univariate general linear modelling was employed.

The following terms were initially included in all models: sire, sex, rearing rank (litter size), maternal selection line (fat or lean), slaughter order (the first animal to be slaughtered on a given day is assigned rank 1 , the next is assigned rank 2, etc.) and date of birth. All terms were factorial except slaughter order and date of birth, which were fitted as covariates. Initially, models were constructed with all terms fitted as both main effects and first-order interactions. Statistical significance of each term was assessed by $\mathrm{F}$ ratios. A minimal model was constructed by dropping all terms that were not significant at $P<0.05$. The minimal model was then used as a baseline model, to which genetic terms ( $f$ or MLH) were added. Both genetic terms were initially fitted as main effects and as interactions with sire. Note that terms containing $f$ and MLH were not fitted in the same model. A significant interaction term would indicate betweensire variation in the number of segregating partially deleterious recessive alleles. The nine traits that were measurable in both sexes were positively correlated with each other (all correlations $P<0.001$ ), so multivariate analysis of variance (MANOVA) was also employed. Statistical analyses were implemented in S-plus 6.0 (Insightful, Seattle, WA, USA).

\section{Application of the model to other data sets}

In addition to making a comparison between predictions from the model and observations in an extensive QTL mapping data set, we also examined the likely relationship between $f$ and MLH in a number of other wild and domestic populations. This analysis was restricted to populations for which the mean and variance of $f$ had been estimated, and for which descriptions of microsatellite marker variability were available. The analysis may not be exhaustive, but it does include a number of the best-known vertebrate populations for which inbreeding depression has been reported.

\section{Results}

\section{Predictions from the model}

We first focus on the question of whether MLH is a good predictor of $f$. From equation (4), it can be seen that the correlation between these two variables is a function of the mean and variance of $f$, and of the variance in $H$. The variance in $H$ is itself dependent on the number of loci typed (see equations (1) and (2)). Given the above, an attempt was made to parameterise the model with realistic estimates of mean $(f)$ and $\sigma^{2}(f)$. A literature review identified a number of studies for which these statistics were reported (in a few cases the authors were contacted to obtain $\sigma^{2}(f)$ ). The review included most of the types of population that are frequently the focus of inbreeding depression studies, including island populations (Soay sheep, red deer, song sparrows, collared flycatchers, Darwin's finches), captive populations (wolves), re-introductions (Arabian oryx) and domestic organisms (Coopworth sheep, Lipizzan horses). Details of each study population and $f$ summary statistics are reported in Table 1 . Note that microsatellite markers have been typed in every species. Mean $f$ ranged from a minimum of 0.002 (collared flycatchers) to 0.103 (wolves) - a 50-fold difference. The variance in $f$ ranged from 0.0005 (collared flycatchers) to 0.0192 (wolves) - a 38-fold difference.

Using these parameter estimates, the relative importance of mean $(f), \sigma^{2}(f)$ and the number of typed loci on the correlation coefficient $r(H, f)$ was examined (see Figure $1 \mathrm{a}-\mathrm{c}$ ). Generally, the relationship between $f$ and $H$ was weak, especially for values of mean $(f)$ and $\sigma^{2}(f)$ that are most commonly observed in wild and domestic 
Table 1 Predicted relationship between inbreeding coefficient $(f)$ and MLH in 12 vertebrate populations

\begin{tabular}{|c|c|c|c|c|c|c|c|c|}
\hline Species & Mean $(f)$ & $\sigma^{2}(f)$ & $\mathrm{n}$ & Mean $\mathrm{h}_{e}$ & $\mathrm{~L}$ & $r(H, f)_{e}$ & $r(H, f)_{o}$ & $r(H, f)_{100}$ \\
\hline Coopworth sheep (Ovis aries) & 0.052 & 0.0008 & 590 & 0.72 & $138(101)$ & $-0.46(-0.39)$ & -0.17 & -0.38 \\
\hline Soay sheep (Ovis aries) & $0.007^{a}$ & 0.0009 & 898 & $0.66^{\mathrm{g}}$ & 14 & -0.16 & & -0.42 \\
\hline Bighorn sheep (Ovis canadensis) & $0.015^{*}$ & 0.0020 & 107 & 0.66 & 20 & -0.27 & -0.15 & -0.56 \\
\hline Red deer (Cervus elaphus) & $0.019^{a}$ & 0.0025 & 553 & $0.76^{\mathrm{i}}$ & 9 & -0.25 & & -0.60 \\
\hline Arabian oryx (Oryx leucoryx) & $0.041^{\mathrm{a}}$ & 0.0066 & 122 & $0.42^{j}$ & 6 & -0.18 & & -0.77 \\
\hline Wolves (Canis lupus) & $0.103^{\mathrm{b}}$ & 0.0192 & 30 & $0.66^{\mathrm{k}}$ & 29 & $-0.71^{\dagger}$ & -0.72 & -0.90 \\
\hline Lipizzan horses (Equus caballus) & $0.101^{\mathrm{c}}$ & 0.0007 & 360 & $0.67^{c}$ & 17 & -0.15 & -0.03 & -0.36 \\
\hline Collared flycatchers (Ficedula albicollis) & $0.002^{\mathrm{d}}$ & 0.0005 & 2107 & $0.82^{1}$ & 3 & -0.08 & & -0.32 \\
\hline Song sparrows (Melospiza melodia) & $0.051^{\mathrm{e}}$ & 0.0041 & 285 & $0.63^{\mathrm{m}}$ & 6 & -0.22 & & -0.69 \\
\hline Large ground finch (Geospiza magrinostris) & $0.070^{\mathrm{h}}$ & 0.0143 & 76 & $0.66^{\mathrm{h}}$ & 14 & -0.56 & -0.54 & -0.87 \\
\hline Medium ground finch (Geospiza fortis) & $0.010^{\mathrm{f}, \S}$ & 0.0004 & 212 & $0.635^{\mathrm{n}, \S}$ & 13 & $-0.10^{\dagger}$ & -0.20 & -0.30 \\
\hline Cactus finch (Geospiza scandens) & $0.042^{\mathrm{f}, \S}$ & 0.0044 & 75 & $0.607^{\mathrm{n}, \S}$ & 13 & $-0.28^{\dagger}$ & -0.04 & -0.70 \\
\hline Median & 0.042 & 0.0023 & 249 & 0.66 & 13.5 & -0.21 & -0.17 & -0.58 \\
\hline Mean & 0.042 & 0.0047 & 451 & 0.65 & 20.4 & -0.27 & -0.26 & -0.57 \\
\hline
\end{tabular}

Mean $(f)$ and $\sigma^{2}(f)$ were estimated from $n$ individuals in each population. All grandparents were known for every individual (often substantially more pedigree information was available). Each population has also been typed for $L$ microsatellite loci with mean heterozygosity $h_{\mathrm{e}}$. This information is then used to estimate the correlation coefficient between $f$ and MLH: $r(H, f)$. Where available, the observed correlation is also reported: $r(H, f)_{0}$. Finally, the predicted correlation coefficient between $f$ and $H$ assuming 100 loci with mean heterozygosity 0.7 is also reported: $r(H, f)_{100}$. Unless otherwise stated, $\sigma^{2}(H)$ was estimated from equation $(1)$. Estimated and observed $r(H, f)$ values for Coopworth sheep are for 138 and 101 loci (see Methods).

a Marshall et al (2002), 'bedrick et al (2001), 'Curik et al (2003), 'Kruuk et al (2002), 'Keller (1998), 'Keller et al (2002), gColtman et al (1999), ${ }^{\mathrm{h}}$ Grant et al (2001), 'Marshall et al (1998), iMarshall and Spalton (2000), kVila et al (2003), 'Sheldon and Ellegren (1996), mJeffery et al (2001), nPetren (1998), *DW Coltman, P O’Donoghue and M Festa-Bianchet (personal communication), \$J Market, K Petren, L Keller, BR Grant and PR Grant (personal communication), ‘variance in $H$ estimated from equation (2).

vertebrate populations. The relationship was largely insensitive to mean $(f)$ (see Figure 1a), but was very dependent on $\sigma^{2}(f)$ (see Figure 1b). This result is unsurprising - no relationship is expected in a population where all individuals have the same $f$, regardless of the actual value of $f$. Note that when 10 loci are typed the correlation between $f$ and $H$ is always weak $(r<0.5)$. For populations with $\sigma^{2}(f)<0.005$ (nine out of 12 populations considered here meet this criterion), a genome scan of 200 highly variable markers would only produce crude estimates of individual $f$. In summary, unless the study population has very high variance in $f, M L H$ is only weakly correlated to $f$.

\section{Analysis of the Coopworth sheep data set}

Individual inbreeding coefficient was positively skewed and possibly bimodally distributed (Figure 2a), while MLH followed an approximately normal distribution (Figure 2b). $f$ had a mean of 0.052 and a variance of 0.008 (Table 1). In comparison with the other 11 populations reported in Table 1, this gave Coopworth sheep the fourth largest mean $f$ and the ninth largest variance in $f$. Thus, the population is not unusual in terms of observed levels of inbreeding. Standardised MLH was highly correlated with its unstandardised equivalent $(r=0.984)$.

Relationship between heterozygosity and inbreeding coefficient: MLH was significantly and negatively correlated with $f(r=-0.177 ; P<0.0001$; Figure 3$)$. However, the correlation coefficient between the two variables was weak, despite MLH being measured at a far larger number of loci than any similar study. In fact, the observed correlation coefficient between $f$ and $H$ was considerably weaker than that predicted under the model (predicted $r=-0.39$ ). Using equation (3a), the expected correlation coefficient between $f$ and MLH has a standard deviation of 0.038 , such that the observed $r(H$, $f)$ was 5.6 standard deviations lower than expected $(P<0.001)$.

Is heterozygosity correlated across the genome?: For marker heterozygosity to provide an estimate of heterozygosity at functionally important loci, it should also be correlated between individual marker loci. We calculated the correlation coefficients between heterozygosity at pairs of individual loci - for example, the correlation between heterozygosity at locus $a$ and at locus $b$. The process was repeated for every pair of loci and the sign of the correlation noted. A standard sign test cannot be used to determine whether there were significantly more positive than negative correlations than expected by chance, because not every correlation is independent. Instead, we used the randomisation approach described by Slate and Pemberton (2002). There were more negative correlations (4653) than there were positive correlations (4630), and there were not significantly more positive correlations than expected by chance $(P=0.331$; based on 10000 permutations). However, feeding the observed values of marker heterozygosity, mean $f$ and $\sigma^{2}(f)$ into equation (3), it becomes clear that the power of this test is weak. The expected correlation in heterozygosity between two markers is only 0.0045, with a standard deviation almost 10-fold greater (0.0412). In other words, a negative correlation is expected to be observed almost as often as a positive correlation.

An alternative solution to the problem is to pool together all of the correlations between the 138 loci within one test. The sum of the covariances in heterozygosity between each pair of loci can be calculated as (Lynch and Walsh, 1998)

$$
2 \sum_{i<j}^{n} \sigma\left(h_{i}, h_{j}\right)=\sigma^{2}\left(\sum_{i} h_{i}\right)-\sum_{i} \sigma^{2}\left(h_{i}\right)
$$



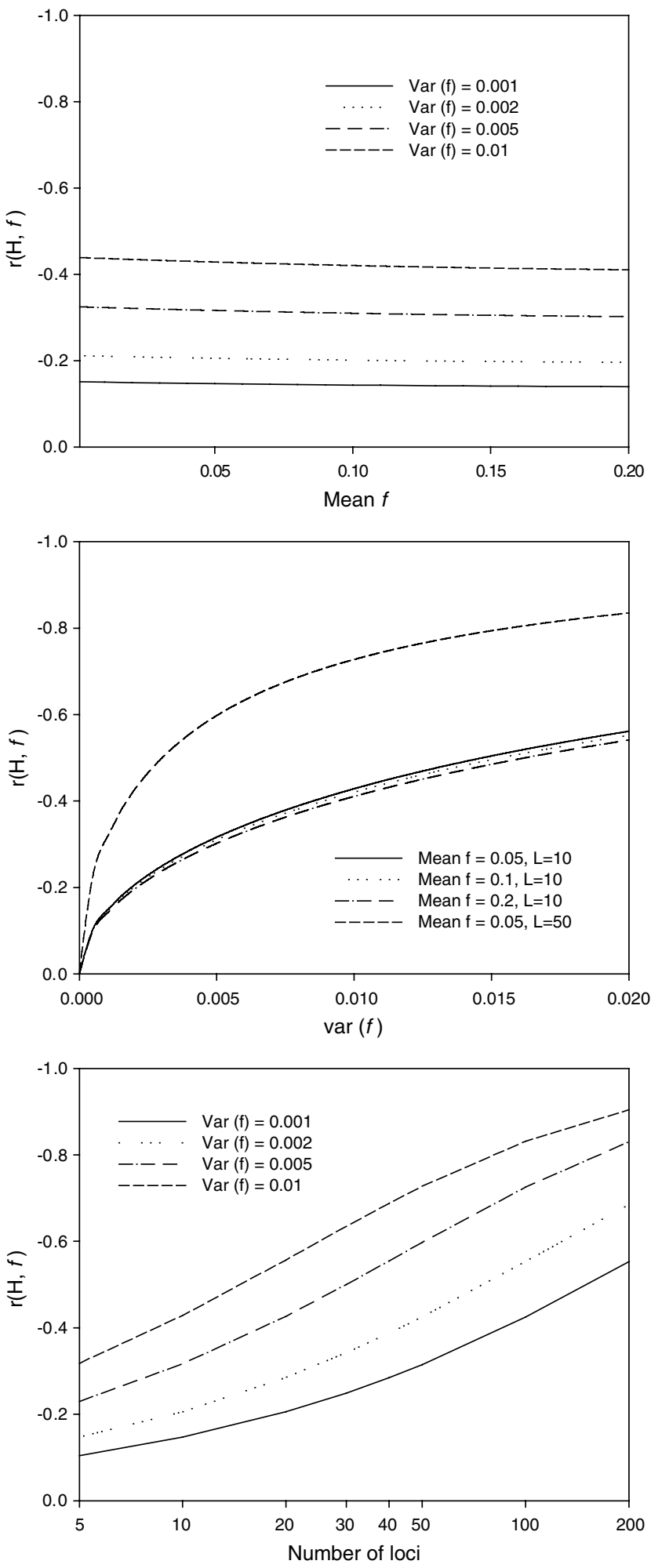

Figure 1 Estimated correlation coefficient between MLH $(H)$ and inbreeding coefficient $(f)$ as a function of (a) mean $f,(\mathbf{b})$ the variance in $f$ and (c) the number of loci typed $(L)$. It is assumed that each locus has a mean heterozygosity of 0.7 . Unless otherwise stated, it is assumed that each individual is typed at 10 loci.

where $h_{i}$ is individual heterozygosity at locus $i$ and $h_{j}$ is individual heterozygosity at locus $j$, and each individual is typed at $n=138$ loci, yielding $\sigma\left(h_{i}, h_{j}\right)=0.51$. Note that missing values were replaced by the locus population
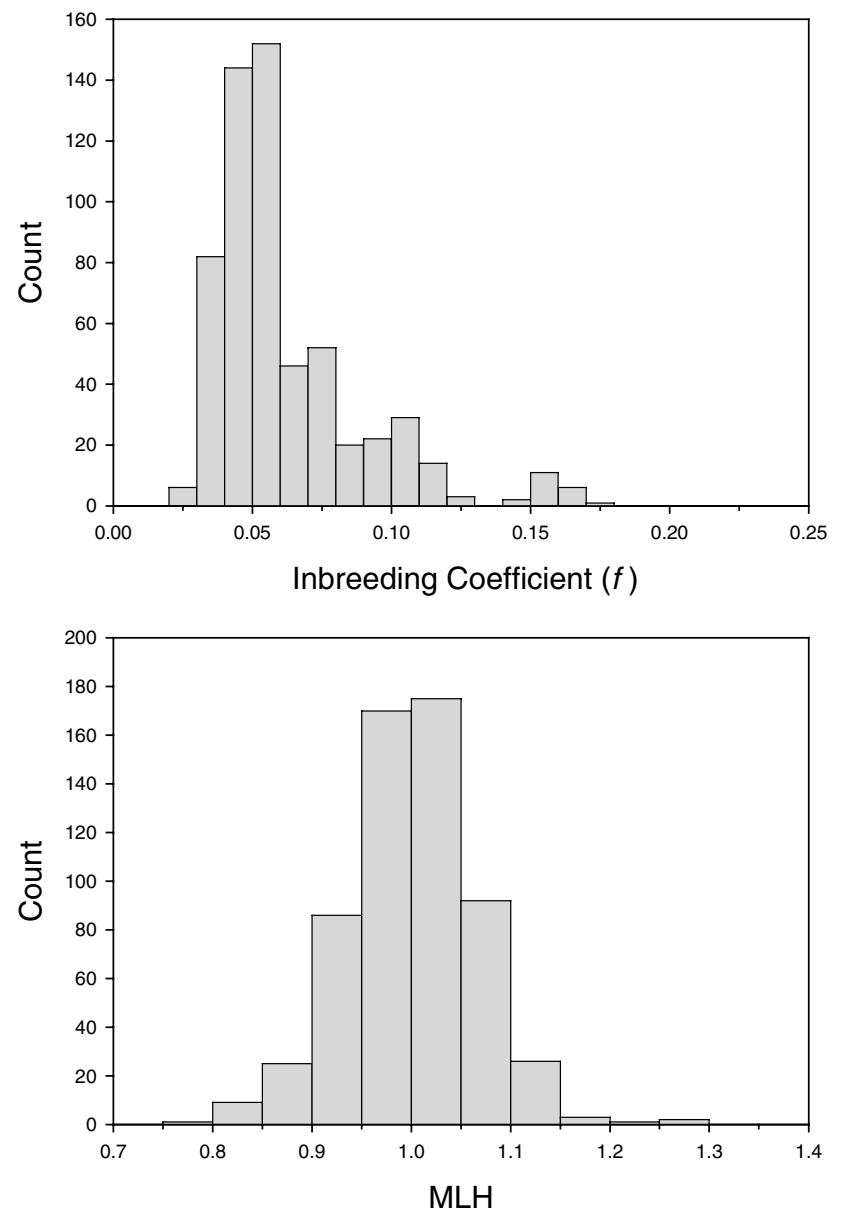

Figure 2 Distribution of (a) individual inbreeding coefficients, (b) individual MLH among 590 Coopworth sheep.

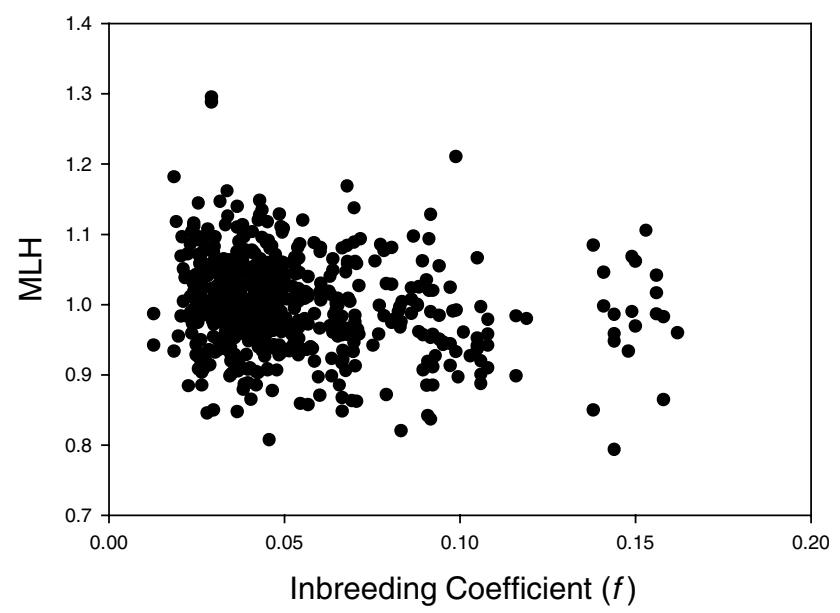

Figure 3 Relationship between individual inbreeding coefficient and MLH in 590 Coopworth sheep.

mean, providing a conservative estimate of the test statistic. To determine the statistical significance of the test statistic, individual heterozygosity was permuted across individuals (sampled without replacement) at each locus, and $\sigma\left(h_{i}, h_{j}\right)$ was recalculated. This process was repeated 10000 times, and the actual test statistic was not significant $(P=0.178)$. In summary, 


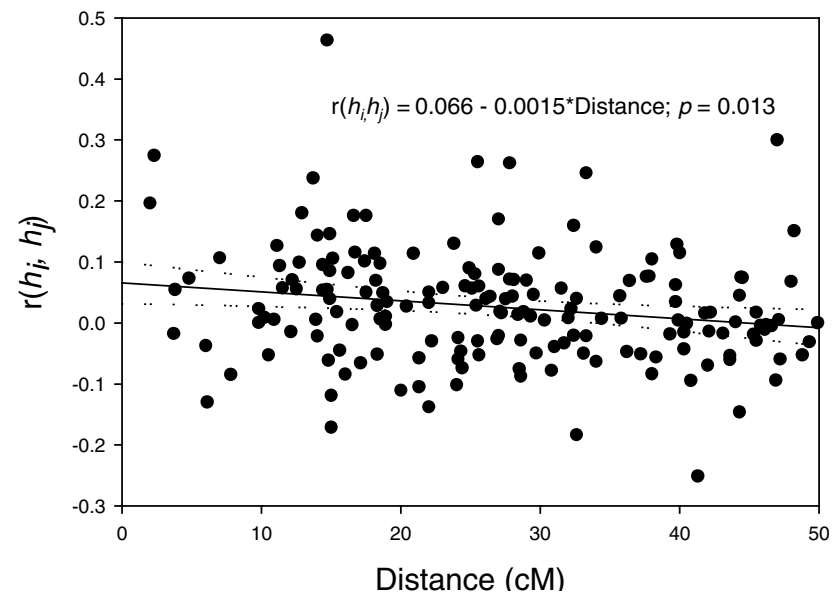

Figure 4 Relationship between $r\left(h_{i}, h_{j}\right)$ and distance (in Kosambi centiMorgans) for linked pairs of microsatellites that map within $50 \mathrm{cM}$ of each other.

heterozygosity is not correlated across the genome, indicating that in this population marker heterozygosity would not accurately reflect heterozygosity at unlinked functionally important loci.

For the purposes of this paper, loci are regarded as linked if they map within $50 \mathrm{cM}$ of each other (alleles at linked loci separated by greater distances can be regarded as independently segregating). Among linked pairs of loci, heterozygosity was more often positively correlated than negatively correlated (104 positive versus 68 negative correlations; $P=0.003 ; 1000$ permutations of the data). Furthermore, the correlation in heterozygosity was a function of the Kosambi centiMorgans distance between loci; $r\left(h_{i}, h_{j}\right)=0.066-0.0015 \times$ distance, $P=0.013$, $n=172, r^{2}=0.036$ (Figure 4). Thus, individual marker heterozygosity does appear to be an indicator of heterozygosity at linked loci.

Do $\mathrm{MLH}$ or $f$ explain variation in morphological traits?: Univariate analyses did not reveal significant associations between any morphological trait and $f$ fitted as a main effect. However, when fitted as an interaction term with sire, $f$ explained significant variation in the following traits: empty body weight $\left(\mathrm{F}_{4,551}=4.19\right.$, $P=0.002)$; hot carcass weight $\left(\mathrm{F}_{4.552}=4.96, P<0.001\right)$; spleen weight $\left(\mathrm{F}_{4,556}=4.85, \quad P<0.001\right)$; liver weight

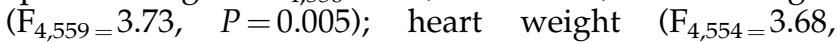
$P=0.006)$; tibia length $\left(\mathrm{F}_{4,554=2.94, P=0.020) \text {; carcass }}\right.$

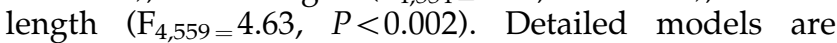
presented in Table 2. For all traits, the association was largely driven by a strong effect observed in the half-sib progeny of sire 616, with relatively inbred animals being generally smaller. These data suggest that, relative to the other four sires, sire 616 carries more deleterious recessive alleles at loci that influence growth traits. The significant relationship between $f$ and morphometric variation among the progeny of sire 616 is not attributable to outlying data points. The two terms, $f$ and $f \times$ Sire, together explained between $0.75 \%$ (backfat depth) and $2.80 \%$ (spleen weight) of the variation in any trait.

MLH was not a significant term in any univariate analysis, whether fitted as a main effect or as an interaction term with sire. In summary, $f$, but not MLH, revealed inbreeding depression for morphometric traits.

The relationship between $f$ and morphometric variation was also revealed by the multivariate analysis. The interaction between sire and $f$ was a significant term when fitted in the MANOVA of the nine morphometric traits $\left(F_{36,2208}=1.451, P=0.041\right)$ but $f$ fitted as a main effect was not $\left(\mathrm{F}_{9,549}=0.903, P=0.523\right)$. MLH was not a significant term either as a main effect $\left(F_{9,549}=1.5\right.$, $P=0.144)$ or as an interaction with sire $\left(F_{36,2208}=0.715\right.$, $P=0.896)$. Note that $f$ and MLH were not fitted in the same model for either the univariate or the multivariate analyses.

\section{Predictions for other vertebrate populations}

In addition to the Coopworth sheep population, summary statistics relating to $f$ and marker heterozygosity were collected for 11 other populations. These data were then used to estimate the correlation coefficient between $f$ and MLH (a) with the markers that have been typed in the study population to date, and (b) if 100 markers of mean heterozygosity 0.7 were typed. Estimates are presented in Table 1. The population for which MLH was the best predictor of $f$ was Scandinavian wolves with an expected $r(H, f)=-0.71$ if the 29 documented microsatellites were typed and an expected $r(H, f)=$ -0.90 if 100 loci were typed. The population for which MLH was worst at predicting $f$ was the collared flycatchers (Ficedula albicollis) on the Swedish Island of Gotland, with an expected $r(H, f)=-0.08$ if the three documented microsatellites were typed and an expected $r(H, f)=-0.32$ if 100 loci were typed. Generally, heterozygosity would not provide robust estimates of $f$, even when 100 loci are typed. For example, the expected $r(H, f)$ is weaker than -0.5 for five of the 12 populations and weaker than -0.7 for nine of the populations.

In seven of the populations, $r(H, f)$ had actually been estimated, enabling a comparison between expected and observed correlation coefficients (Table 1). In Scandinavian wolves and Large Ground Finches, the observed and expected correlation coefficients were almost identical. In four of the five other populations, $r(H, f)_{\text {observed }}$ was weaker than $r(H, f)_{\text {expected, perhaps due to errors in }}$ estimation of $f$ (see Discussion).

\section{Discussion}

The primary objective of this study was to establish if and when MLH can be used as a robust surrogate for individual $f$. A theoretical model and empirical data both suggest that the correlation between MLH and $f$ is weak unless the study population exhibits unusually high variance in $f$. The Coopworth sheep data set used in this study comprised a considerably larger number of genotypes (590 individuals typed at 138 loci) than any similar study, yet MLH was only weakly correlated to individual $f$. Furthermore, $f$ explained significant variation in a number of morphometric traits (typically 1-2\% of the overall trait variance), but heterozygosity did not. From equation (5), it can be seen that the expected correlation between trait value and MLH is the product of the correlation coefficient between $f$ and the trait (hereafter $r(W, f)$ ) and $r(H, f)$. Estimates of the proportion of phenotypic trait variation explained by $f$ are scarce, although from the limited available data $2 \%$ seems a 
Table 2 Univariate analysis of 10 morphometric traits examined in a population of Coopworth sheep

\begin{tabular}{|c|c|c|c|c|c|c|c|c|c|c|c|}
\hline Term & $d f$ & $E B W(k g)$ & $H C W(k g)$ & Heart $(g)$ & Liver $(g)$ & Spleen $^{\dagger}(g)$ & Tibia $(\mathrm{mm})$ & Carcass length $(\mathrm{cm})$ & Testicles (g) & Longissimus dorsi (kg) & Backfat depth ( $\mathrm{mm}$ \\
\hline \multicolumn{12}{|l|}{ Minimal model } \\
\hline$n$ & & 573 & 573 & 573 & 573 & 573 & 573 & 573 & 266 & 573 & 573 \\
\hline Mean & & 54.8 & 27.3 & 242 & 819 & 85 & 210.0 & 109.2 & 456 & 0.99 & 23.0 \\
\hline SD & & 9.4 & 5.2 & 39.6 & 139.7 & 21.6 & 10.3 & 5.5 & 94.8 & 0.25 & 8.7 \\
\hline Sex & 1 & $440.99 * * *$ & $187.34^{* * *}$ & $245.09^{* * *}$ & $540.31^{* * *}$ & $25.11^{* * *}$ & $118.79^{* * *}$ & $406.34^{* * *}$ & - & 0.04 & $81.85^{* * *}$ \\
\hline Maternal line & 2 & $20.28^{* * *}$ & $3.29^{*}$ & $22.77^{* * *}$ & $16.03^{* * *}$ & $34.83^{* * *}$ & $39.03^{* * *}$ & $64.34^{* * *}$ & $28.29 * * *$ & $7.94^{* * *}$ & $76.25^{* * *}$ \\
\hline Sire & 4 & $17.60^{* * *}$ & $18.72^{* * *}$ & $5.43^{* * *}$ & $12.63^{* * *}$ & $8.87^{* * *}$ & $2.79 *$ & $72.78^{* * *}$ & $5.81^{* * *}$ & $8.99^{* * *}$ & $11.72^{* * *}$ \\
\hline Rearing rank & 1 & - & $6.33^{*}$ & $4.59^{*}$ & $8.75^{* * *}$ & - & - & $5.00^{*}$ & - & - & - \\
\hline Slaughter order & 1 & - & - & 0.74 & - & 0.01 & - & - & - & $54.00^{* * *}$ & - \\
\hline Birth date & 1 & $5.39^{*}$ & $8.22^{* *}$ & - & - & - & - & - & - & $6.21^{*}$ & $9.20^{* *}$ \\
\hline Sire $\times$ Sex & 4 & $4.21^{* *}$ & $3.62^{* *}$ & - & - & - & $2.55^{*}$ & - & - & $4.14^{* *}$ & - \\
\hline Sire $\times$ Line & 4 & - & - & - & - & - & - & - & - & - & $3.31^{* *}$ \\
\hline Sire $\times$ Order & 4 & - & - & $3.34^{*}$ & - & - & - & - & - & - & - \\
\hline Sex $\times$ Order & 2 & - & - & - & - & $9.33^{* *}$ & - & - & - & - & - \\
\hline Line $\times$ RearRk & 2 & $4.47^{*}$ & - & - & - & - & - & - & - & - & - \\
\hline Line $\times$ Sex & 2 & - & - & - & - & - & - & - & - & - & $4.43^{*}$ \\
\hline \multicolumn{12}{|c|}{ With inbreeding terms } \\
\hline$f$ & 1 & 0.81 & 0.27 & 0.52 & 0.16 & 2.10 & 0.11 & 0.02 & 0.88 & 0.45 & 2.17 \\
\hline$f$-Sire & 4 & $4.19^{* *}$ & $4.99^{* * *}$ & $3.68^{* *}$ & $3.73^{* *}$ & $4.85^{* * *}$ & $2.94^{*}$ & $4.63^{* *}$ & 0.70 & 2.37 & 1.98 \\
\hline Residual SS & & 18562 & 6520 & 472641 & 5204909 & 4.354 & 41535 & 6987 & 1800682 & 22.82 & 17664 \\
\hline$f \%$ ss & & 1.15 & 1.54 & 1.45 & 1.26 & 2.80 & 1.27 & 1.35 & 1.24 & 1.13 & 0.75 \\
\hline MLH & 1 & 2.05 & 0.62 & 0.18 & 0.02 & 0.02 & 1.33 & 0.00 & 0.05 & 1.49 & 0.04 \\
\hline MLHSire & 4 & 0.47 & 0.36 & 0.36 & 0.21 & 1.39 & 1.35 & 1.25 & 1.05 & 0.25 & 1.80 \\
\hline \multicolumn{12}{|l|}{ Coefficients (SE) } \\
\hline$f$ & & $-5.18(9.32)$ & $-0.77(5.57)$ & $50.16(47.26)$ & $-14.85(155.56)$ & $0.27(0.14)$ & $-2.46(14.11)$ & $0.74(5.70)$ & $-86.21(202.87)$ & $-0.12(0.33)$ & $-11.26(9.50)$ \\
\hline$f \times 608$ & & $-9.16(15.76)$ & $-6.09(9.40)$ & $-55.89(79.87)$ & $-86.66(263.49)$ & $-0.38(0.24)$ & $-17.74(23.63)$ & $-2.83(9.66)$ & $-136.97(348.13)$ & $-0.48(0.56)$ & $-3.76(15.98)$ \\
\hline$f \times 610$ & & $-0.93(8.53)$ & $-1.62(5.10)$ & $-8.03(43.11)$ & $-24.77(141.96)$ & $-0.18(0.13)$ & $19.25(12.91)$ & $3.11(5.20)$ & $210.97(202.00)$ & $-0.15(0.30)$ & $-4.01(8.88)$ \\
\hline$f \times 616$ & & $-23.20(5.75)$ & $-14.78(3.44)$ & $-109.47(29.06)$ & $-363.50(95.84)$ & $-0.30(0.09)$ & $-29.08(8.72)$ & $-14.55(3.51)$ & $-151.81(114.22)$ & $-0.57(0.20)$ & $-13.68(5.74)$ \\
\hline$f \times 620$ & & $2.02(4.74)$ & $2.82(2.78)$ & $8.68(23.59)$ & $43.21(77.40)$ & $-0.18(0.07)$ & $10.12(7.04)$ & $1.59(2.84)$ & $-77.23(97.00)$ & $0.16(0.16)$ & $6.07(4.68)$ \\
\hline MLH & & $4.41(4.34)$ & $1.53(2.59)$ & $-20.19(22.23)$ & $-10.24(72.59)$ & $-0.04(0.07)$ & $-13.22(6.52)$ & $-1.12(2.68)$ & $-72.19(94.47)$ & $0.18(0.15)$ & $4.54(4.34)$ \\
\hline
\end{tabular}

EBW $=$ empty body weight, $\mathrm{HCW}=$ hot carcass weight. For a detailed description of the model, see the Methods section. The F statistic of each significant term in the minimal model is reported,

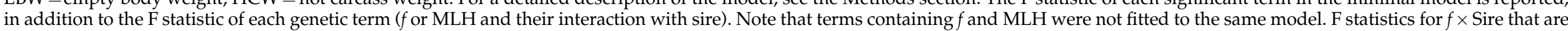

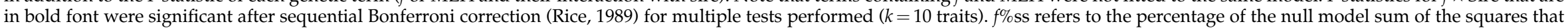

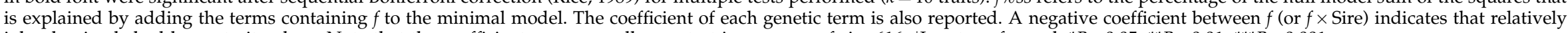
inbred animals had lower trait values. Note that the coefficient was generally greatest in progeny of sire 616 . ${ }^{*}$ Log transformed. ${ }^{*} P<0.05 ;{ }^{* *} P<0.01 ;{ }^{* * *} P<0.001$ 
typical value (see for example Kruuk et al, 2002; this paper, Table 2). Assuming $r(W, f)^{2}=0.02$, and given the median value of $r(H, f)=-0.21$ reported in Table 1 , a crude estimate of average $r(W, H)$ is 0.03 , which is equivalent to MLH explaining $<0.1 \%$ of trait variance. These findings are consistent with a recent meta-analysis that reported a mean $r(W, H)$ of 0.09 for life history traits and 0.01 for morphometric traits (Coltman and Slate, 2003). In summary, MLH is a poor replacement for $f$, such that very large sample sizes are required to detect variance in inbreeding in most populations.

If MLH has limited power to detect variance in inbreeding, what is the most parsimonious explanation for studies reporting HFCs? One possibility is that marker loci are in physical linkage with loci that determine trait variation, such that detected HFCs are attributable to local effects rather than a general effect caused by variance in inbreeding. In fact, general and local effects reflect the same phenomenon, that is, the existence of deleterious recessives and/or overdominant alleles dispersed throughout individual genomes. Heterozygosity at marker loci will always be more correlated to autozygosity at closely linked fitness loci than at unlinked loci, as linkage is known to increase identity disequilibria. However, depending on how many fitness loci are segregating in a population, and on population structure and history, the contribution of the chromosomal vicinity of a given marker to HFC may or may not be negligible compared to that of the rest of the genome. Models of general effects (such as the above model) assume (i) that inbreeding depression is homogeneously distributed throughout the genome, rather than concentrated in a few loci, and (ii) that all loci are unlinked. When one or both of these conditions are seriously violated, general effect models predict less HFC than there actually is, as local effects take more importance. In such conditions, we would also expect that the contributions of different marker loci to HFC become significantly different, and that linked markers tend to behave in the same way with regard to HFC. It should be noted that although $4 \%$ of our loci were linked, only $0.11 \%$ of locus pairs were separated by a distance of less than $10 \mathrm{cM}$. Furthermore, the maximum correlation in heterozygosity observed between any pair of loci was very low $(r=0.01)$, indicating that each of the 138 loci can be regarded as an independent sample of heterozygosity.

Although no association was detected between MLH and trait variation in Coopworth sheep, it is notable that heterozygosity was only correlated between linked loci and that the correlation declined as a function of physical distance (Figure 4). Thus, if an association had been detected, it could potentially be attributable to a local effect. Among studies reporting significant HFCs, a recent analysis of Great Reed Warblers (Acrophalus aruninaceus) provides the best evidence for local effects being the underlying mechanism (Hansson et al, 2001). The Great Reed Warbler experiment maximised the probability of detecting local effects, as it was conducted within pairs from the same brood (hence each member of a pair had the same $f$, and general effects were excluded). The more heterozygous member of a pair had greater probability of recruiting to the adult population. Elsewhere, local effects and identity disequilibrium were found to explain simultaneously an association between birth weight and MLH at 71 microsatellites typed in red deer (Cervus elaphus). MLH was positively and significantly associated with birth weight, and heterozygosity was correlated across loci (Slate and Pemberton, 2002). However, heterozygosity at two individual loci explained additional variation in birth weight after MLH at the remaining loci was fitted to the model. The two loci were subsequently shown to be physically linked to birth weight QTL on two separate chromosomes (Slate et al, 2002). Thus, local effects have been demonstrated to be a cause of some HFCs. Note that the studies we refer to concern only vertebrates, the main source of pedigreed data sets. The mating systems of vertebrates (obligate biparental reproduction, frequent postnatal dispersal) may leave less opportunity to generate a high variance in inbreeding, than exists in other organisms such as molluscs or self-fertile plants, in which HFC has traditionally been observed. It may be that vertebrate populations are especially favourable situations in which to observe local effects.

It is also clear that there is a publication bias in favour of HFCs of greatest magnitude (Coltman and Slate, 2003). Often HFC studies are conducted simply because the marker data are available, for example, after microsatellites were typed to examine population genetic structure or for parentage analysis. There may be a tendency for spurious associations to be reported in the literature and presented as evidence for inbreeding depression. Significant HFCs are only likely to be caused by inbreeding depression if $\sigma^{2}(f)$ or $r(W, f)$ are large. Alternatively, those studies that do reveal a significant association may represent the low proportion of experiments expected to generate a significant test statistic despite a lack of power. Therefore, it seems reasonable to conclude that any attempt to infer inbreeding depression via variance in MLH is likely to end in failure even when very large numbers of individuals or markers (or both) are typed. Furthermore, those experiments that do reveal significant HFCs usually reveal little information about the underlying mechanism, and in the absence of additional support do not provide evidence of inbreeding depression.

Overall, the model was a good predictor of the observed correlation between $f$ and $\operatorname{MLH}(r(H, f)$ observed $=$ $\left.0.11+1.06 r(H, f)_{\text {expected }} r^{2}=0.78, P<0.01 ; \mathrm{df}=5\right)$. There was a trend for $r(H, f)_{\text {observed to be weaker than }}$

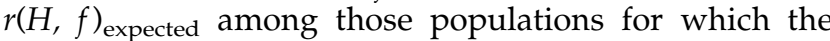
comparison was possible. The most likely explanation for this trend is that the pedigrees contained some errors, resulting in errors in estimated $f$. Alternatively, the founder animals in each pedigree had nonzero values of $f$ or were related. Under either scenario, individual $f$ inferred from the pedigree may be inaccurate, adding

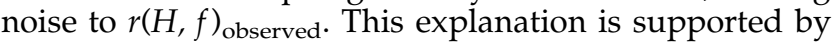
the observation that the Scandinavian Wolf and Large Ground Finch populations provided very similar estimated and observed correlation coefficients. The wolves were from a captive population that has been closely managed and is small, making pedigree errors unlikely. Furthermore, the eight founder animals came from four geographical locations (two animals per location) so that each founder can safely be assumed to be unrelated to at least six of the seven other founders (Hedrick et al, 2001). The Finch population was recently founded by a small number of immigrant birds, making accurate pedigree construction relatively straightforward. In contrast, the 
other populations were all large or were not intensively managed, making inaccuracies in estimated $f$ more likely. Given that observed and estimated correlations were only available for seven populations, it seems prudent to avoid drawing more solid conclusions at this stage.

One area of future investigation that might be addressed is to examine whether alternative microsatellite-based variables are superior indicators of $f$. One measure, mean $d^{2}$, has received recent attention, although theoretical (Tsitrone et al, 2001), empirical (Slate and Pemberton, 2002) and meta-analytical (Coltman and Slate, 2003) studies suggest that this metric is even less useful than heterozygosity. A more promising alternative is the use of methods that estimate the relatedness of an individual's parents from the focal individual's genotype (Ritland, 1996; Lynch and Ritland, 1999; Amos et al, 2001). The potential advantage of such an approach is that allele frequency is incorporated into the measure; for example, an individual homozygous for a rare allele is more likely to be inbred than an individual homozygous for a common allele. Unfortunately, the Coopworth sheep microsatellites were scored in such a way as to make such an analysis impossible.

For the purposes of this analysis, the Coopworth sheep data set was chosen for its magnitude (with respect to the number of animals and loci typed), rather than as an attempt to better understand the genetics of production traits in sheep per se. However, the observed inbreeding depression and/or heterosis in this population is not surprising or unprecedented. Inbreeding depression for morphological traits in other breeds has been described elsewhere (Falconer, 1989; Wiener et al, 1992). Furthermore, the two lines from which these sheep were derived showed an asymmetric response towards selection for backfat depth (Morris et al, 1997), an observation consistent with a trait having a relatively large dominance variance component (Falconer, 1989; Frankham, 1991; Merilä and Sheldon, 1999), and so being susceptible to inbreeding depression.

To conclude, a theoretical model and an extensive data set suggest that MLH is a poor indicator of $f$ even in populations where inbreeding is common. These findings are consistent with previous investigations that have failed to detect significant HFCs in large, randomly mating populations (Houle, 1989; Savolainen and Hedrick, 1995) or in structured populations (Whitlock, 1993). Furthermore, it is apparent that marker heterozygosity does not always provide a robust estimate of genome-wide heterozygosity but may reflect heterozygosity at linked loci. These issues should always be considered when attempting to detect inbreeding depression in populations for which pedigree records are unavailable.

\section{Acknowledgements}

We thank Gordon Greer, Wendy Bain and Neville Jopson for maintenance and measurement of the resource flocks. Dave Coltman, Lukas Keller, Jeffrey Markert and Loeske Kruuk provided useful summary statistics for their study populations. Anna Campbell, Josephine Pemberton and an anonymous referee made useful comments on an earlier version of the manuscript.

\section{References}

Acevedo-Whitehouse K, Gulland F, Greig D, Amos W (2003). Disease susceptibility in California sea lions. Nature 422: 35.

Allendorf FW, Leary RF (1986). Heterozygosity and fitness in natural populations of animals. In: Soule ME (ed) Conservation Biology, Sinauer: Sunderland, MA. pp 57-76.

Amos W, Worthington Wilmer J, Fullard K, Burg TM, Croxall JP, Bloch D et al (2001). The influence of parental relatedness on reproductive success. Proc R Soc Lond B 268: 2021-2027.

Bierne N, Tsitrone A, David P (2000). An inbreeding model of associative overdominance during a population bottleneck. Genetics 155: 1981-1990.

Campbell AW, Bain WE, McRae AF, Broad TE, Johnstone PD, Dodds KG et al (2003). Bone density in sheep: genetic variation and quantitative trait loci (QTL) localisation. Bone 33: $540-548$.

Charlesworth B, Charlesworth D (1999). The genetic basis of inbreeding depression. Genet Res 74: 329-340.

Coltman DW, Pilkington JG, Smith JA, Pemberton JM (1999). Parasite-mediated selection against inbred Soay sheep in a free-living, island population. Evolution 53: 1259-1267.

Coltman DW, Slate J (2003). Microsatellite measures of inbreeding: a meta-analysis. Evolution 57: 971-983.

Curik I, Zechner P, Solkner J, Achmann R, Bodo I, Dove P et al (2003). Inbreeding, microsatellite heterozygosity, and morphological traits in Lipizzan horses. J Hered 94: 125-132.

David P (1998). Heterozygosity-fitness correlations: new perspectives on old problems. Heredity 80: 531-537.

Grant PR, Grant BR, Petren K (2001). A population founded by a single pair of individuals: establishment, expansion, and evolution. Genetica 112-113: 359-382.

Falconer D (1989). Introduction to Quantitative Genetics, Longman: New York.

Frankham R (1991). Are responses to artificial selection for reproductive fitness characters consistently asymmetrical? Genet Res 56: 35-42.

Hansson B, Bensch S, Hasslequist D, kesson M (2001). Microsatellite diversity predicts recruitment of sibling great reed warblers. Proc R Soc Lond B 268: 1287-1291.

Hansson B, Westerberg L (2002). On the correlation between heterozygosity and fitness in natural populations. Mol Ecol 11: 2467-2474.

Hartl DL, Clark AG (1997). Principles of Population Genetics, Sinauer: Sunderland, MA.

Hedrick P, Fredrickson R, Ellegren H (2001). Evaluation of d2, a microsatellite measure of inbreeding and outbreeding, in wolves with a known pedigree. Evolution 55: 1256-1260.

Hedrick PW, Kalinowski ST (2000). Inbreeding depression in conservation biology. Annu Rev Ecol Syst 31: 139-162.

Houle D (1989). Allozyme associated heterosis in Drosophila melanogaster. Genetics 123: 789-801.

Jeffery KJ, Keller LF, Arcese P, Bruford MW (2001). The development of microsatellite loci in the song sparrow, Melospiza melodia (Aves) and genotyping errors associated with good quality DNA. Mol Ecol Notes 1: 11-13.

Keller LF (1998). Inbreeding and its fitness effects in an insular population of sparrows (Melospiza melodia). Evolution 52: 240-250.

Keller LF, Grant PR, Grant BR, Petren K (2002). Environmental conditions affect the magnitude of inbreeding depression in survival of Darwin's Finches. Evolution 56: 1229-1239.

Keller LF, Waller DM (2002). Inbreeding effects in wild populations. Trends Ecol Evol 17: 230-240.

Kruuk LEB, Sheldon BC, Merilä J (2002). Severe inbreeding depression in collared flycatchers (Ficedula albicollis). Proc $R$ Soc Lond B 269: 1581-1589.

Lynch M, Ritland K (1999). Estimation of pairwise relatedness with molecular markers. Genetics 152: 1753-1766.

Lynch M, Walsh B (1998). Genetics and Analysis of Quantitative Traits, Sinauer: Sunderland, MA. 
Marshall TC, Coltman DW, Pemberton JM, Slate J, Spalton JA, Guinness FE, Smith JA, Pilkington JG, Clutton-Brock TH (2002). Estimating the prevalence of inbreeding from incomplete pedigrees. Proc $R$ Soc Lond B 269: 1533-1539.

Marshall TC, Slate J, Kruuk LEB, Pemberton JM (1998). Statistical confidence for likelihood-based paternity inference in natural populations. Mol Ecol 7: 639-655.

Marshall TC, Spalton JA (2000). Simultaneous inbreeding and outbreeding depression in reintroduced Arabian oryx. Anim Conserv 3: 241-248.

Merilä J, Sheldon BC (1999). Genetic architecture of fitness and nonfitness traits: empirical patterns and development of ideas. Heredity 83: 103-109.

Mitton JB (1993). Theory and data pertinent to the relationship between heterozygosity and fitness. In: Thornhill NW (ed) The Natural History of Inbreeding and Outbreeding, University of Chicago Press: Chicago.

Morris CA, McEwan JC, Fennessy PF, Bain WE, Greer GJ, Hickey SM (1997). Selection for high or low backfat depth in Coopworth sheep: juvenile traits. Anim Sci 65: 93-103.

Morton NE, Crow JF, Muller HJ (1956). An estimate of the mutational damage in man from data on consanguineous matings. Proc Natl Acad Sci USA 42: 855-863.

Petren K (1998). Microsatellite primers from Geospiza fortis and cross-species amplification in Darwin's finches. Mol Ecol 7: 1782-1784.

Rice WR (1989). Analyzing tables of statistical tests. Evolution 43: 223-225.

Ritland K (1996). A marker-based method for inferences about quantitative inheritance in natural populations. Evolution 50: 1062-1073.

Savolainen O, Hedrick P (1995). Heterozygosity and fitness: no association in scots pine. Genetics 140: 755-766.
Sheldon BC, Ellegren H (1996). Offspring sex and paternity in the collared flycatcher. Proc $R$ Soc Lond B 263: 1017-1021.

Slate J, Kruuk LEB, Marshall TC, Pemberton JM, Clutton-Brock TH (2000). Inbreeding depression influences lifetime breeding success in a wild population of red deer (Cervus elaphus). Proc $R$ Soc Lond B 267: 1657-1662.

Slate J, Pemberton JM (2002). Comparing molecular measures for detecting inbreeding depression. J Evol Biol 15: $20-31$.

Slate J, Visscher PM, MacGregor S, Stevens DR, Tate ML, Pemberton JM (2002). A genome scan for QTL in a wild population of red deer (Cervus elaphus). Genetics 162: 1863-1873.

Thornhill NW (ed) (1993). The Natural History of Inbreeding and Outbreeding, University of Chicago Press: Chicago.

Tsitrone A, Rousset F, David P (2001). Heterosis, marker mutational processes and population inbreeding history. Genetics 159: 1845-1859.

Vila C, Sundqvist AK, Flagstad O, Seddon J, Bjornerfeldt $S$ Kojola I, Casulli A, Sand H, Wabakken P, Ellegren H (2003). Rescue of a severely bottlenecked wolf (Canis lupus) population by a single immigrant. Proc $R$ Soc Lond $B$ 270: 91-97.

Weir BS, Cockerham CC (1973). Mixed self and random mating at two loci. Genet Res Camb 21: 247-262.

Whitlock M (1993). Lack of correlation between heterozygosity and fitness in forked fungus beetles. Heredity 70: 574-581.

Wiener G, Lee GJ, Wooliams JA (1992). The effects of rapid inbreeding and of crossing of inbred lines on the body weight growth of sheep. Anim Prod 55: 89-99.

Wright $S$ (1922). Coefficients of inbreeding and relationship. Am Nat 56: 330-339.

Zar JH (1996). Biostatistical Analysis, Prentice-Hall: London. 